\title{
BMJ Open Gonadotropin-releasing hormone agonist versus expectant management for treating multiple leiomyomas after myomectomy: the study protocol for a multicentre, prospective, randomised controlled clinical trial
}

Jia Wei (D) , ${ }^{1}$ Xiangyi Ma, ${ }^{1}$ Wenwen Wang, ${ }^{1}$ Minli Zhang, ${ }^{1}$ Zhiying Yu, ${ }^{2}$ Wei Zhang, ${ }^{3}$ Li Hong, ${ }^{4}$ Zhiying Li, ${ }^{5}$ Lin Li, ${ }^{6}$ Xin Du, ${ }^{7}$ Yun Feng, ${ }^{8}$ Ruixia Guo, ${ }^{9}$ Chunlian Zhang, ${ }^{10}$ Qingfen Yue, ${ }^{11}$ Wuliang Wang, ${ }^{12}$ Shixuan Wang ${ }^{1}$

To cite: Wei J, Ma X, Wang W, et al. Gonadotropin-releasing hormone agonist versus expectant management for treating multiple leiomyomas after myomectomy: the study protocol for a multicentre, prospective, randomised controlled clinical trial. BMJ Open 2021;11:e044347. doi:10.1136/ bmjopen-2020-044347

- Prepublication history for this paper is available online. To view these files, please visit the journal online (http://dx.doi org/10.1136/bmjopen-2020044347).

Received 01 September 2020 Accepted 28 July 2021

Check for updates

(C) Author(s) (or their employer(s)) 2021. Re-use permitted under CC BY-NC. No commercial re-use. See rights and permissions. Published by BMJ.

For numbered affiliations see end of article.

Correspondence to Professor Shixuan Wang; shixuanwang@tjh.tjmu.edu.cn

\section{ABSTRACT}

Introduction Leiomyoma recurrence is a major concern for long-term myomectomy management, especially for multiple leiomyomas. Gonadotropin-releasing hormone agonist ( $\mathrm{GnRHa}$ ) is one of the most effective medications to reduce the volume of fibroids and the uterus. However, its role in preventing recurrence after conservative surgery remains unclear. At present, there is no randomised clinical trial determining the efficacy of GnRHa treatment for preventing multiple leiomyomas recurrence after myomectomy.

Methods and analysis We are conducting a phase IV randomised controlled trial in women aged 18-45 undergoing myomectomy for multiple leiomyomas. After surgery, women whose pathological result confirms multiple leiomyomas are randomised in a 1:1 ratio into an observation or GnRHa group. The primary outcome is the recurrence of either clinical symptoms or fibroids on imaging. Patients will be assessed for adverse events during the follow-up.

Ethics and dissemination The study was approved by the Medical Ethics Committee of the Tongji Hospital Affiliated with the Tongji Medical College of Huazhong University of Science and Technology (TJ-IRB20180311) according to the submitted study protocol (V.1.0, 10 November 2017) and informed consent (V.1.0, 10 November 2017). The results will be presented at domestic and international conferences and published in peerreviewed journals.

Trial registration number ChiCTR-IPR-17012992.

\section{INTRODUCTION}

Leiomyoma, also called myoma or uterine fibroid, is the most common benign uterine tumour among women of reproductive age. Its clinical presentations include a pelvic mass, chronic pelvic pain, abnormal uterine bleeding and infertility. ${ }^{1}$ Leiomyoma occurs in $50 \%-60 \%$ of women of reproductive age,
Strengths and limitations of this study

This is the first randomised controlled trial investigating the efficacy of a gonadotropin-releasing hormone agonist for preventing high-risk leiomyoma recurrence after myomectomy.

- This is a large-scale, multiple centres, prospective study.

- The pictorial blood loss assessment chart and imaging will be employed to evaluate multiple leiomyomas symptoms.

Data on the recurrent time and rate will be collected.

- The hypogonadal side effects may lead to drop-outs during the study.

rising to $70 \%$ by age $50,{ }^{2}$ translating into 880 million women worldwide. It originates from the smooth muscle cells in the myometrium and is highly dependent on oestrogen stimulation. Multiple uterine fibroids are a special form of leiomyoma, referring to more than one type of myoma in the uterus with a much higher recurrence risk than a single leiomyoma. ${ }^{34}$

Currently, multiple leiomyoma treatment involves primarily surgical interventions. However, the surgical approach is largely dependent on the patients' age and desire to preserve fertility. In Asian culture, patients are prone to preserving the organ's integrity and, therefore, myomectomy is more popular among the Asian population. Gynaecologists are also increasingly realising that preserving the uterus can help maintain the blood supply to the ovaries, maintaining ovarian function. Therefore, myomectomy has become the dominant choice. After myomectomy, close 
follow-ups are recommended. However, it is widely known that the leiomyoma recurrence rate after myomectomy can be as high as $60 \%$ after 5 years ${ }^{56}$ and even higher for multiple leiomyomas. ${ }^{5}$

The oestrogen level heavily influences leiomyoma growth. Thus, starting in the 1980s, gonadotropinreleasing hormone agonists (GnRHa) were investigated as a potential treatment. Studies have confirmed that GnRHa decreases the volume of fibroids and the uterus. ${ }^{78}$ Currently, GnRHa is recommended preoperatively, as it reduces preoperative and perioperative blood loss. ${ }^{9}$ However, clinical investigations regarding its ability to prevent multiple leiomyomas recurrence after conservative surgery are lacking.

At present, there is no guideline for long-term multiple leiomyoma management. A randomised trial is warranted to test the efficacy of GnRHa for preventing multiple leiomyoma recurrence. This study is a multicentre, prospective, randomised controlled trial aiming to determine the efficacy of GnRHa compared with expectant management for recurrence prevention among women who underwent myomectomy.

We believe that the proposed trial is significant because it evaluates the effectiveness of GnRHa treatment for preventing multiple leiomyoma recurrence, which is one of the most common benign tumours among reproductive-age women. Further, it will provide evidence for long-term multiple leiomyoma management after myomectomy.

\section{METHODS AND ANALYSIS \\ Overall design}

This study is designed to compare the multiple leiomyoma recurrence rate after a myomectomy with and without GnRHa treatment. We will recruit patients aged 18-45 years diagnosed with multiple leiomyomas and undergoing myomectomy. Eligible and willing participants will be randomised to a either GnRHa treatment or observational group in a 1:1 ratio. The GnRHA treatment will be six intramuscular injections of $3.75 \mathrm{mg}$ of GnRHa administered once per month. Participants will be followed for 36 months after enrolment. The assessment will be recorded by a masked interviewer at 3, 6, 12, 18, 24, 30 and 36 months after enrolment. The primary outcomes will be the leiomyoma recurrence. Bias will be minimised by a rigorous randomisation procedure and by having interviewers masked to the participant's treatment group.

\section{Study population}

This study is being conducted in 12 academic hospitals located in central China.

\section{Sample size calculation}

Based on the preliminary retrospective data from our department, the multiple leiomyoma recurrence rate 3 years after surgery is approximately $30 \%$. We assumed that an absolute difference of $10 \%$ in the recurrence rate is clinically significant. Therefore, the number needed to treat is 100 . We aim to test a $10 \%$ difference in the recurrence rate between the treatment and observation groups (ie, 20\% in the GnRHa group and $30 \%$ in the observation group) at a significance level of 0.05 with a statistical power of $80 \%$. The minimum calculated sample size is 291 per group. Considering a 10\% drop-out rate, we will enrol 320 subjects per group.

\section{Inclusion criteria}

The inclusion criteria are:

1 . Women aged $\geq 18$ and $\leq 45$ years.

2. Women diagnosed with multiple leiomyomas, which is more than one type of leiomyoma in the uterus, including subendometrial, intramural and subserosal leiomyoma.

3. Women undergoing myomectomy and have histopathologically confirmed leiomyoma.

4. One month after the surgery, the ultrasound must confirm no fibroid or the fibroid diameter is smaller than $2 \mathrm{~cm}$.

5. Women who have not used leiomyoma medications within 3 months before the enrolment.

6 . Women who can give written consent.

\section{Exclusion criteria}

The exclusion criteria are:

1. Women diagnosed with congenital uterine abnormalities, such as a uterine malformation (eg, a unicornuate uterus, septate uterus or duplex uterus).

2. Pregnant women.

3. Patients with inadequate fibroid removal, defined as residual fibroids larger than $2 \mathrm{~cm}$ in the ultrasound scan 1 month after myomectomy.

4. Women diagnosed with other oestrogen dependent diseases, such as adenomyoma or endometriosis.

5. Women who used a GnRHa treatment within 3 months before enrolment.

6. Women with medical conditions that contraindicate surgery, such as uncontrolled hypertension or known symptomatic heart disease, poorly controlled type 1 or type 2 diabetes mellitus, undiagnosed liver disease or dysfunction (based on a serum liver enzyme test), renal disease or abnormal serum renal function, severe anaemia, a history of deep venous thrombosis, pulmonary embolus or cerebrovascular accident, a history of (or suspected) cervical carcinoma, endometrial carcinoma or breast carcinoma.

7. Women allergic to the GnRHa treatment.

8. Women simultaneously undertaking other clinical trials.

\section{Screening and enrolment}

At the screening visit, the medical history and the current medication status will be recorded with the standardised case report forms. Laboratory measurements, including basal sex hormone tests, the anti-müllerian hormone 
$(\mathrm{AMH})$ level, safety assays (eg, liver function and renal function), antibodies for hepatitis virus, HIV and syphilis, coagulation and routine blood and urine tests were performed in the local labs of the study sites. The symptoms are assessed using the Short-Form Health Survey (SF-36) and pictorial blood loss assessment chart (PBAC).

The patients will undergo myomectomy for multiple leiomyomas, and the histopathology report must confirm leiomyoma. Myomectomy can be conducted by the laparoscopic, hysteroscopic or abdominal approach. The surgery length, numbers and size of the fibroids, the blood loss volume and the uterine cavity integrity will be recorded. Imaging, such as transvaginal ultrasound scan or MRI, will be performed 1 month after the surgery to confirm that there are no remaining fibroids or the fibroid diameter is smaller than $2 \mathrm{~cm}$.

After the initial screening, the clinicians will approach potential participants, introduce the study, explain the current treatment progression and invite participation. The patients will be aware of the randomised assignment for the treatment group. Written consent will be obtained from the patients after a thorough discussion. The enrolled population will include all patients who provided informed consent.

\section{Randomisation and blinding}

Subjects will be assigned to two groups with a 1:1 ratio using simple randomisation. All centres except for one (the main centre) have the same scale regarding inpatient number and surgery volume. Therefore, a simple randomisation is suitable for this study. The estimated recruitment rate is 12 cases in the main centre and 6 cases in the other centres per month. The randomisation sequence was generated by biostatisticians in a data coordinator centre using Microsoft Excel. The original sequence is kept secure and is input into the online central randomisation system by the data coordinator centre staff members who are not involved in subject enrolment. The online sequence is not accessible to any investigators or study coordinators. If a subject fulfils the enrolment criteria, the authorised study coordinator will get the assignment for the subject. The patient's name will be replaced by a sequence number to maintain confidentiality. After randomisation, the patients and clinicians will be informed about the assignments.

During the follow-up, the investigator will be blinded to the participant's treatment assignment to minimise reporting bias. The data will be collected and uploaded to an online data storage application as it develops.

\section{The intervention}

Participants in the treatment group will receive one $3.75 \mathrm{mg}$ GnRHa intramuscular injection (Diphereline, Debiopharm, Ipsen, France) on menstruation day 1 after the surgery. The intervention will be repeated monthly for 6 months. The rationale for six GnRHa courses is that suppression is the greatest at 6 months, and the side effects are manageable at this time. Since the patients underwent myomectomy, pregnancy is not recommended for 18 months. Therefore, six GnRHa courses will not interfere with the patients' pregnancy plan. A Standard Protocol Items: Recommendations for Interventional Trials flow diagram of enrolment, interventions and assessment is provided in figure 1 .

\section{Follow-up checks and interviews}

All enrolled participants will be checked and interviewed according to the follow-up schedule outlined in figure 1 . There will be seven follow-ups after the surgery, once every 3 months for the first two visits, then once every 6 months for the remaining visits. During each visit, oestradiol (E2), follicle-stimulating hormone (FSH) and AMH levels will be tested, and a pelvic ultrasound will be performed. The blood samples will be destroyed after

\begin{tabular}{|c|c|c|c|c|c|c|c|c|c|c|}
\hline \multirow[b]{3}{*}{ TIMEPOINT } & \multicolumn{10}{|c|}{ STUDY PERIOD (months) } \\
\hline & \multirow{2}{*}{$\begin{array}{c}\text { Enrolment } \\
-1\end{array}$} & \multirow{2}{*}{$\begin{array}{c}\text { Allocation } \\
0\end{array}$} & \multicolumn{7}{|c|}{ Post-allocation } & \multirow{2}{*}{$\frac{\text { Close-out }}{36}$} \\
\hline & & & 3 & 6 & 12 & 18 & 24 & 30 & 36 & \\
\hline \multicolumn{11}{|c|}{ ENROLMENT } \\
\hline \multirow{3}{*}{$\begin{array}{c}\text { Eligibility screen } \\
\text { Informed consent } \\
\text { Allocation }\end{array}$} & $x$ & & & & & & & & & \\
\hline & & $x$ & & & & & & & & \\
\hline & & $x$ & & & & & & & & \\
\hline \multicolumn{11}{|c|}{ INTERVENTIONS } \\
\hline \multirow{2}{*}{$\begin{array}{c}\text { GnRHa } \\
\text { Expectant therapy }\end{array}$} & & $\leftarrow$ & & $\longrightarrow$ & & & & & & \\
\hline & & & & & & & & & & \\
\hline \multicolumn{11}{|c|}{ ASSESSMENTS } \\
\hline \multirow{3}{*}{$\begin{array}{c}\text { Ultrasound/MRI } \\
\text { SF-36/PBAC } \\
\text { FSH, E2, AMH }\end{array}$} & $x$ & $x$ & $x$ & $x$ & $x$ & $x$ & $X$ & $x$ & $x$ & \\
\hline & $x$ & & $x$ & $x$ & $x$ & $x$ & $x$ & $x$ & $x$ & \\
\hline & $x$ & & $x$ & $\mathrm{X}$ & $x$ & $\mathrm{x}$ & $x$ & $x$ & $x$ & \\
\hline
\end{tabular}

Figure 1 The SPIRIT flow diagram of the study illustrating the enrolment, intervention and assessment of this project. AMH, anti-müllerian hormone; FSH, follicle-stimulating hormone; GnRHa, gonadotropin-releasing hormone agonist; PBAC, pictorial blood loss assessment char; SF-36, Short-Form Health Survey. 
laboratory evaluation. The SF-36 and PBAC results, the medication history and any side effects will be recorded. If there is a withdrawal, the investigator will record the time and reason for the drop-out.

\section{Data collection}

Patients will be recruited from March 2018 to September 2021, and the follow-up will be completed over the 3 years after surgery. One allocated investigator is in charge of recording follow-ups, and this investigator will be blinded to the participant's treatment assignment. The data will be collected with a standard case report form and uploaded to an online data storage application developed by a third party. Data will be deidentified before uploading into the online recording platform. Regular study site monitoring and database checking will be performed to ensure data collection accuracy. A data monitoring committees is not set up in this study as this is a phase IV trial.

The principal investigator will have the access to the final dataset on the online data storage application.

\section{Patient and public involvement}

Patients or the public were not involved in the design, or conduct, or reporting, or dissemination plans of our research.

\section{Data analysis}

\section{Safety assessment}

Adverse events (AE) are any unfavourable medical occurrence associated with the subject's participation in the research, whether or not it is related to the study intervention. All potential AEs will be monitored throughout the trial with supervision from the Huazhong University of Technology and Science Ethnic committee. Participants can quit the trial at any time if there is an intolerance to the therapy.

Hypogonadal side effects from GnRHa treatment, such as hot flashes, anxiety and bone loss, should be carefully evaluated and may be treated with add-back therapy to maintain E2 levels at $30-50 \mathrm{pg} / \mathrm{mL}$.

AEs unrelated to GnRHa will also be recorded and assessed by investigators. A severe $\mathrm{AE}$ (SAE) is any reaction that led to the discontinuation of the medication owing to intolerable side effects, which can sometimes be fatal. The AE severity will be assessed by the attending investigator, who will also decide on the discontinuation of the medication.

\section{Primary outcomes}

The primary outcome is the recurrence time and the recurrence rate. Recurrence is the return of symptoms or fibroid growth. The symptoms are increased menstruation blood loss (ie, a PBAC score greater than 100) and fibroid growth, which is a fibroid diameter larger than $2 \mathrm{~cm}$ or the number increases compared with the baseline image 1 month after the surgery.

\section{Secondary outcomes}

The secondary outcomes are comparisons between the treatment and observational groups: (1) The proportions of participants with AEs, (2) pregnancy outcomes and (3) the quality of life after the myomectomy assessed by SF-36 questionnaire, the ovarian reservation (ie, AMH, E2 and FSH levels) and the uterine size.

\section{Data analysis}

Data analysis and reporting will be conducted following the Consolidated Standards of Reporting Trials guidelines (recorded in the flow chart, figure 1) and include the number of eligible participants and the number lost to follow-up for various reasons.

The relevant medication used after enrolment will be recorded by the interviewer in each follow-up interview. The use of combined oral contraceptive will be considered a variable in the analysis. Intention to treat will be used as the foundation in our analysis and includes all enrolled patients. The per-protocol population will include all participants who met the inclusion and exclusion criteria, received a full course of the GnRHa treatment and completed the primary relevant visits. This population excludes patients with major protocol deviations, such as those not completing all six GnRHa courses or using other sex hormonal devices.

Baseline characteristic comparisons will be performed between the observational and intervention groups. Continuous data will be analysed by Wilcoxon rank-sum test and reported as means and SD. Categorical data will be analysed by Pearson's $\chi^{2}$ test and reported as numbers and percentages.

The primary outcome measure is the recurrence rate difference between the observational and intervention groups after 3 years of follow-up, which will be analysed by Pearson's $\chi^{2}$ test. For efficacy parameters, such as the menstruation blood loss score, the uterus size and lesions, will be analysed using generalised estimating equations to identify correlations among these observations at different follow-up points.

The secondary outcomes parameters, such as the SF-36 score and the PBAC score, will be calculated during the 3-year follow-up by mixed model for repeated measures analysis to compare differences between the two groups at different time points. The intention-to-treat results will be compared with the per-protocol analysis to provide information on the potential effect of selective lost to follow-up.

The number of participants with AEs or SAEs will be presented for each treatment arm, and the proportion of AEs and SAEs will be analysed as secondary outcomes. A post hoc sensitivity analysis will also be executed to identify the reasons for participants' withdrawal.

\section{ETHICS AND DISSEMINATION}

The experiments outlined in this protocol involve a randomised controlled clinical trial on humans and 
were approved by the Medical Ethics Committee of the Tongji Hospital Affiliated to Tongji Medical College of Huazhong University of Science and Technology (TJIRB20180311) based on the submitted study protocol (V.1.0, 10 November 2017) and informed consent (V.1.0, 10 November 2017). The ethical approval was approved at the central and local levels. The central ethical approval was confirmed by the Medical Ethics Committee of the Tongji Hospital (ref approval no. TJ-IRB20180311), and we will not begin recruiting at other centres in the trial until local ethical approval has been granted.

This study is registered with the Chinese Clinical Trial Registry. The results will be submitted for academic conference presentations and publication in peerreviewed journals, and the conclusions will be presented at each participating centre and shared with the trial participants.

\section{DISCUSSION}

This study compares participants with and without GnRHa intervention after a myomectomy for multiple leiomyomas. We plan to enrol 640 subjects across 12 teaching hospitals in China. The enrolment began in March 2018.

At the time of manuscript preparation, the recruitment is still undergoing. The result of this large multicentre randomised trial will provide level I evidence for a longterm management strategy for multiple leiomyomas after myomectomy.

Currently, the treatment for leiomyoma largely relies on surgery. Hysterectomy is more radical, but it can also cause more short-term and long-term complications. Conversely, myomectomy preserves the uterus's integrity, ${ }^{10}$ but the recurrence rate can be as high as $30 \%$ 3 years after surgery. Repetitive surgery would be more challenging because of the iatrogenic adhesion, and sometimes hysterectomy is inevitable. The aetiology of leiomyoma is still not fully understood. However, epigenetic and metabolic abnormalities have been identified in a large percentage of leiomyomas. ${ }^{11}{ }^{12}$ These could be valuable for developing new therapeutic strategies. ${ }^{13}$ Most leiomyoma medications are based on hormone modulation, such as selective progesterone receptor modulators (SPRM), GnRHa and the combined contraception pill (COP). While COP administration in women aged more than 40 is controversial, the efficacy of SPRMs versus other treatments is also unclear. ${ }^{14} \mathrm{GnRHa}$ was first used to treat leiomyoma in 2010, and using GnRHa preoperatively was confirmed to significantly reduce uterine and fibroid volumes. ${ }^{15}$ However, there remains a lack of clinical trial evidence into the effect of GnRHa for preventing the leiomyoma recurrence after myomectomy, and its role in long-term leiomyoma management is unclear.

Currently, long-term leiomyoma management lacks a standard guideline. We expect this study to provide a reliable answer regarding GnRHa as a preventative intervention for multiple leiomyomas recurrence after myomectomy.

\section{Author affiliations}

${ }^{1}$ Obstetrics and Gynaecology, Tongji Hospital, Tongji Medical College, Huazhong University of Science and Technology, Wuhan, Hubei, China

${ }^{2}$ Obstetrics and Gynaecology, Shenzhen Second People's Hospital, Shenzhen, Guangdong, China

${ }^{3}$ Obstetrics and Gynaecology, Wuhan University Zhongnan Hospital, Wuhan, Hubei, China

${ }^{4}$ Obstetrics and Gynaecology, Wuhan University Renmin Hospital, Wuhan, Hubei,

China

${ }^{5}$ Obstetrics and Gynaecology, Three Gorges University Renhe Hospital, Yichang, Hubei, China

${ }^{6}$ Obstetrics and Gynaecology, Xiangyang Central Hospital, Xiangyang, Hubei, China ${ }^{7}$ Obstetrics and Gynaecology, Maternal and Child Hospital of Hubei Province, Wuhan, Hubei, China

${ }^{8}$ Obstetrics and Gynaecology, First People's Hospital of Yunnan, Kunming, Yunnan, China

${ }^{9}$ Obstetrics and Gynaecology, Zhengzhou University First Affiliated Hospital, Zhengzhou, Henan, China

${ }^{10}$ Obstetrics and Gynaecology, Taihe Hospital, Shiyan, Hubei, China

${ }^{11}$ Obstetrics and Gynaecology, Luoyang Central Hospital Affiliated to Zhengzhou University, Luoyang, Henan, China

${ }^{12}$ Obstetrics and Gynaecology, The Second Affiliated Hospital of Zhengzhou University, Zhengzhou, Henan, China

Acknowledgements We thank all clinicians and patients for participating in this study.

Contributors All authors were involved in the study design. JW and XM contributed to data collection and trial progress management. WeW and $\mathrm{MZ}$ are responsible for the data analysis, ZY, WZ, LH, ZL, LL, XD, YF, RG, CZ, QY and WuW were responsible for participant recruitment in the different centres. SW was responsible for overall trial management.

Funding The authors have not declared a specific grant for this research from any funding agency in the public, commercial or not-for-profit sectors.

Competing interests None declared.

Patient and public involvement Patients and/or the public were not involved in the design, or conduct, or reporting, or dissemination plans of this research.

Patient consent for publication Not required.

Provenance and peer review Not commissioned; externally peer reviewed.

Open access This is an open access article distributed in accordance with the Creative Commons Attribution Non Commercial (CC BY-NC 4.0) license, which permits others to distribute, remix, adapt, build upon this work non-commercially, and license their derivative works on different terms, provided the original work is properly cited, appropriate credit is given, any changes made indicated, and the use is non-commercial. See: http://creativecommons.org/licenses/by-nc/4.0/.

ORCID iD

Jia Wei http://orcid.org/0000-0003-3767-452X

\section{REFERENCES}

1 Donnez J, Jadoul P. What are the implications of myomas on fertility? A need for a debate? Hum Reprod 2002;17:1424-30.

2 Baird DD, Dunson DB, Hill MC, et al. High cumulative incidence of uterine leiomyoma in black and white women: ultrasound evidence. Am J Obstet Gynecol 2003;188:100-7.

3 Radosa MP, Owsianowski Z, Mothes A, et al. Long-term risk of fibroid recurrence after laparoscopic myomectomy. Eur J Obstet Gynecol Reprod Biol 2014;180:35-9.

4 Fauconnier A, Chapron C, Babaki-Fard K, et al. Recurrence of leiomyomata after myomectomy. Hum Reprod Update 2000;6:595-602.

5 Malone LJ. Myomectomy: recurrence after removal of solitary and multiple myomas. Obstet Gynecol 1969;34:200-3.

6 Donnez J, Donnez O, Dolmans MM. With the advent of selective progesterone receptor modulators. what is the place of myoma surgery in current practice? Fertil Steril 2014;102:640-8.

7 Falsetti L, Mazzani MD, Rubessa S, et al. Presurgical treatment of uterine fibroids by using gonadotropin-releasing hormone agonists. Acta Eur Fertil 1992;23:29-32. 
8 Jasonni VM, D'Anna R, Mancuso A, et al. Randomized double-blind study evaluating the efficacy on uterine fibroids shrinkage and on intra-operative blood loss of different length of leuprolide acetate depot treatment before myomectomy. Acta Obstet Gynecol Scand 2001;80:956-8.

9 Zhang Y, Sun L, Guo Y, et al. The impact of preoperative gonadotropin-releasing hormone agonist treatment on women with uterine fibroids: a meta-analysis. Obstet Gynecol Surv 2014;69:100-8.

10 American College of Obstetricians and Gynecologists. ACOG practice bulletin. Alternatives to hysterectomy in the management of leiomyomas. Obstet Gynecol 2008;112:387-400.

11 Holdsworth-Carson SJ, Zaitseva M, Vollenhoven BJ, et al. Clonality of smooth muscle and fibroblast cell populations isolated from human fibroid and myometrial tissues. Mol Hum Reprod 2014;20:250-9.

12 Medikare V, Kandukuri LR, Ananthapur V, et al. The genetic bases of uterine fibroids; a review. J Reprod Infertil 2011;12:181-91.

13 Commandeur AE, Styer AK, Teixeira JM. Epidemiological and genetic clues for molecular mechanisms involved in uterine leiomyoma development and growth. Hum Reprod Update 2015;21:593-615.

14 Murji A, Whitaker L, Chow TL, et al. Selective progesterone receptor modulators (SPRMs) for uterine fibroids. Cochrane Database Syst Rev 2017;4:CD010770.

15 Lethaby A, Puscasiu L, Vollenhoven B. Preoperative medical therapy before surgery for uterine fibroids. Cochrane Database Syst Rev 2017;11:CD000547. 